\title{
A Porcine Liver Model for Validation of Registration Accuracy in Image-guided Surgery
}

\author{
Matthias Peterhans ${ }^{1}$, Benoît Dagon ${ }^{2}$, Anne Vom Berg ${ }^{3}$, Daniel Inderbitzin ${ }^{3}$, \\ Charles Baur ${ }^{2}$, Stefan Weber ${ }^{1}$ \\ ${ }^{1}$ ARTORG Center, University of Bern, Switzerland \\ ${ }^{2}$ VRAI Group, Ecole Polytechnique Fédérale de Lausanne (EPFL), Switzerland \\ ${ }^{3}$ Department of Visceral and Transplantation Surgery, Inselspital, Bern, Switzerland \\ matthias. peterhans@artorg. unibe.ch
}

\begin{abstract}
Correct registration between pre-operative high-resolution images and intra-operative data of lower detail is a fundamental requirement in image-guided liver surgery. We propose a multi modality liver model for measuring the accuracy of such registration methods. A freshly explanted porcine liver is artificially perfused by a peristaltic pump and liver motion is simulated by means of inflatable objects positioned around the liver. Co-registered ultrasound and CT data sets are acquired in different deformation scenarios and allow compar-ing registration outcomes with a CT data set serving as ground truth. The pre-sent work describes the experimental setup and summarizes the results from ultrasound and $\mathrm{CT}$ imaging.
\end{abstract}

\section{Introduction}

In image-guided liver surgery, accurate registration between pre-operative image data and the intra-operative scenario is crucial for correct assistance during the surgery. Registration is either performed based on liver surface data or on information about structures inside the liver. Validation of registration is a challenging task because it is usually difficult to acquire redundant information serving as a benchmark for accuracy computation. Registration success is usually measured by means of the fitting error between corresponding structures (e.g. vessels bifurcations) in the intra-operative data and pre-operative scans [1]. This measure can lead to problems with local minima. Therefore, a measure of registration accuracy by an independent validation data set is desirable.

Related work on liver phantoms reports either synthetic models $[2,3]$ or an ex-vivo human liver model [4]. Bao et al. [2] create liver models with implanted fiducial markers from silicon polymer or polyurethane. Weber et al. [3] use rapid prototyping to print segmented liver structures in an elastic material and then pack the obtained structures in a tissue-mimicking material. Such models allow for multi-modality imaging of the liver (except for Doppler ultrasound) but represent a further abstraction from real liver tissue to tissue mimicking 
material. Müller et al. [4] propose a model based on explanted human livers which are embedded into a respiratory motion simulator for analyzing respiratory motion. Measurements are obtained by navigated needles inserted into the liver parenchyma. Multi modality imaging is mentioned but no results are shown.

In the purpose of staying close to a real surgical situation, we have developed an ex-vivo porcine liver model for multi modality imaging. The experimental setup is built on the table of a CT scanner which allows ultrasound and CT acquisition without motion between the scans. The resulting data sets will serve as ground truth for validating our non-rigid registration approach [5].

\section{Materials and Methods}

\subsection{Methodology}

Our approach to measure the registration accuracy consists of five steps (Figure 1). First, a CT-scan representing the pre-operative situation is acquired (A). Then, the liver is deformed and navigated ultrasound images representing the intra-operative situation are acquired (B). Next, a CT scan of the deformed liver is acquired (C). This CT will serve as the validation data set. Registration between the pre-operative $\mathrm{CT}$ and the intra-operative ultrasound is then performed (D). Finally, the accuracy is measured by calculating the differences between the registered pre-operative $\mathrm{CT}$ and the validation data set $(\mathrm{E})$.

\subsection{Liver preparation}

The proposed liver model consists of an entire porcine liver specimen embedded into foam representing the abdominal organs around the liver. Blood circulation

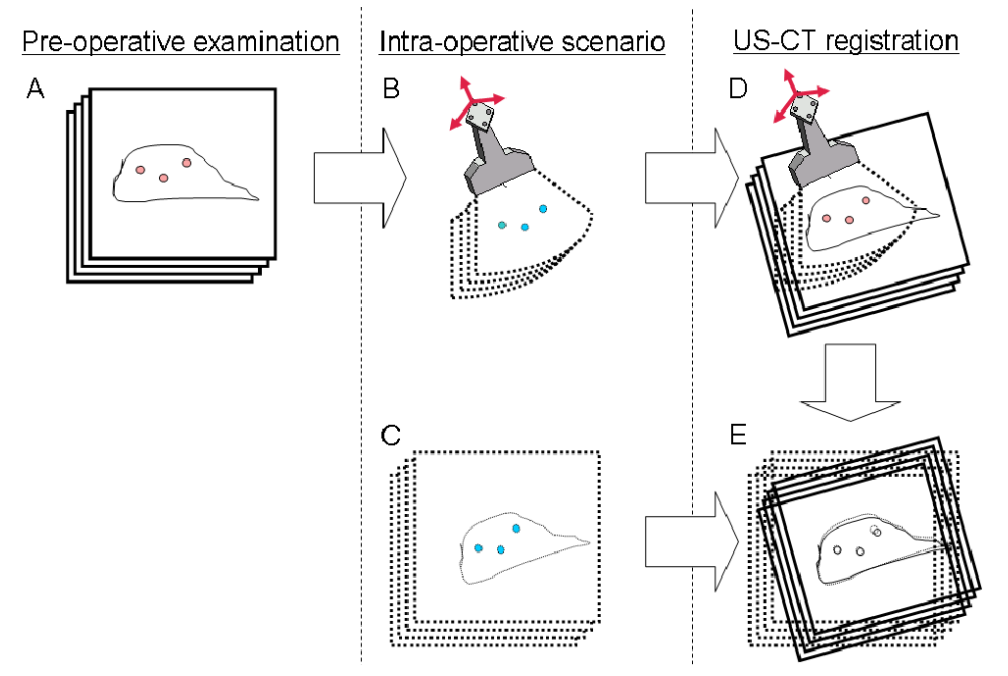

Fig. 1. Illustration of the experimental methodology 
is simulated by a physiological fluid pumped through the liver by a peristaltic pump. The latter is set to provide similar flux and input pressure as observed in the circulatory system. Liver deformation is induced by means of inflatable balloons placed around the liver. Porcine livers were obtained directly from the butcher where care was taken to cut them together with the diaphragm in order not to damage the portal veins. Blood coagulation inside the liver was avoided by rinsing the specimen with Ringer saline solution directly after explantation. The livers where then transported in a cooled bath of $4^{\circ} \mathrm{C}$ of Ringer saline solution and experiments were performed within the same day to minimize the effects of tissue degeneration.

Liver perfusion was induced by a peristaltic pump imposing a flow of Ringer saline solution into the portal vein. Physiologic conditions are obtained when a flow of $200 \mathrm{ml} / \mathrm{min}$ is imposed at the portal vein entrance and the measured input pressure rises to $20 \mathrm{~cm} \mathrm{H} 2 \mathrm{O}$. The perfusion liquid was recollected at the liver veins imposing an exit pressure of $10 \mathrm{~cm} \mathrm{H} 2 \mathrm{O}$.

\section{$2.3 \quad$ Imaging setup}

The liver deformations occurring between the pre-operative CT and the surgery are simulated by means of inflatable balloons positioned around the liver. For each deformation scenario, a co-registered image pair consisting of a CT scan data set and a set of navigated ultrasound images is acquired.

Experiments were performed directly on the table of a Siemens Somatom Emotion 6 CT scanner. Liver vessels were enhanced using Gastrografin (Schering Wien, GmbH) contrast agent mixed with the Ringer saline solution used for perfusion.
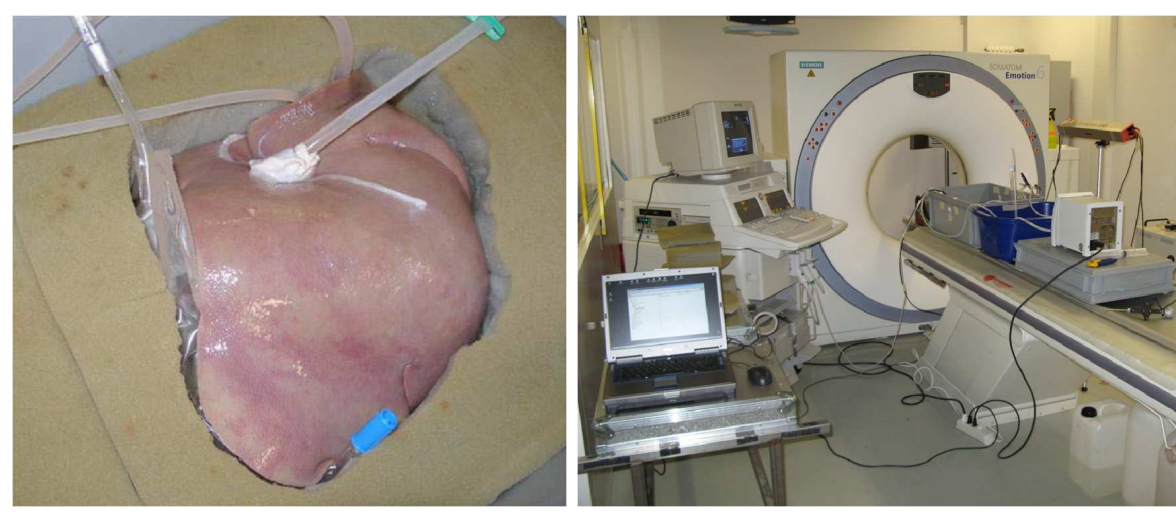

Fig. 2. (Left) Liver specimen with tubes connected to its vascular system. An inflatable bag for simulating deformation is visible on the left. (Right) Experimental setup with ultrasound and CT systems, liver model and pump on the CT table, navigation system. 
After $\mathrm{CT}$ acquisition, ultrasound images were acquired using a calibrated and optically tracked ultrasound probe (Atracsys Easytrack 500 active optical tracking system and Philips Sonos 5500 in 2D mode with an $8 \mathrm{MHz}$ probe) connected to a frame grabber on a notebook computer.

Registration between the CT volume and the navigation system is obtained by pointer-based registration of radiopaque fiducial spheres mounted on the container box.

\section{Results}

For each of the three porcine liver specimens, several pairs of CT-ultrasound image sets were acquired at different deformation scenarios. Figure 3 shows a representative CT image slice and an ultrasound image of a vessel region. In the $\mathrm{CT}$, the main vessels are clearly visible as bright regions. Smaller vessels are still visible but harder to distinguish from the liver parenchyma which also contains bright regions. The latter are due to the continuous contrast injection leading to a permanent filling of liver capillaries. Pulsatile contrast injection as used in clinics would avoid this effect and provide selective enhancement of different structures depending on the delay between contrast injection and image acquisition. However the obtained image quality is sufficient to identify the main liver vessels by semi-automatic segmentation. In the ultra-sound images, vessels are visible as ellipsoidal dark regions which indicates that per-fusion was successfully established and that the imposed pressure simulated physio-logical conditions.

Figure 4 illustrates the results of image processing on the acquired images. The figure on the left shows the superposition of results from semi-automatic segmentation of the pre-operative CT (red surface) and points extracted automatically [5] from the intra-operative ultrasound (red and green circles). On the right, two different deformation stages are visualized using rapid prototyping. Corresponding vessel branches from the pre-operative CT (red) and the validation $\mathrm{CT}$ (blue) are clearly visible.
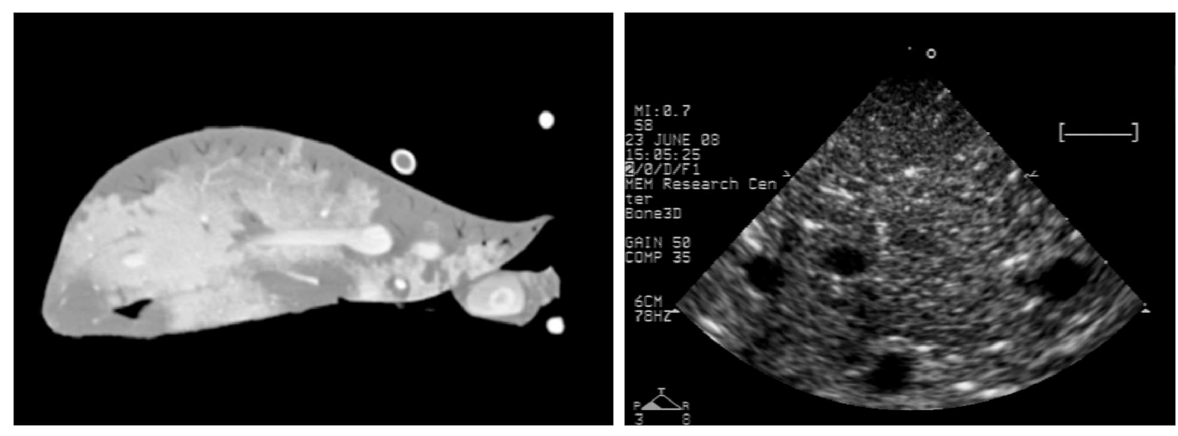

Fig. 3. Results of CT and ultrasound image acquisition on the porcine liver model 
Fig. 4. (Left) Augmented reality environment combining the $3 \mathrm{D}$ vessel model and the ultrasound segmentation results. (Right) 3D print of liver vessels at different deformation stages.
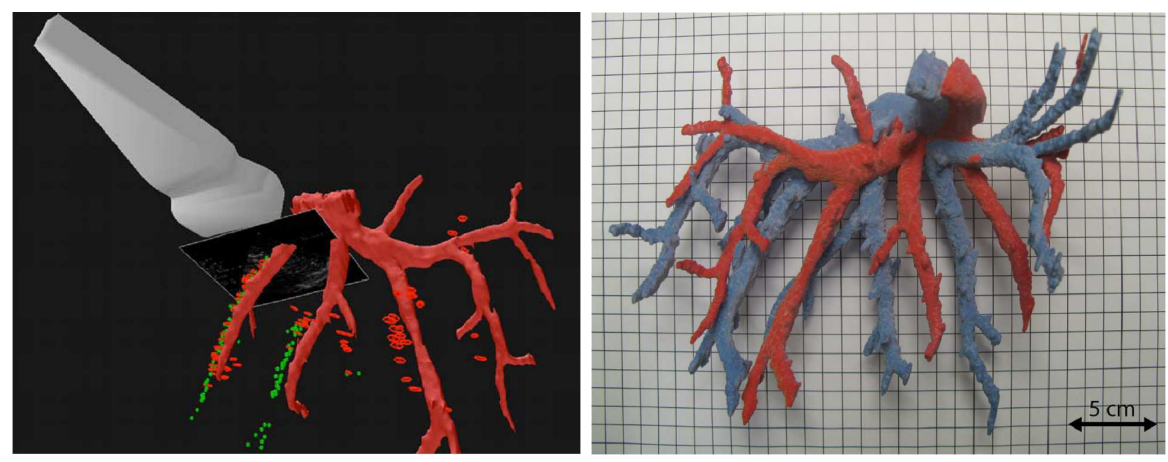

\section{Discussion}

Our experiments have shown the feasibility of multi modality image acquisition on an ex-vivo porcine liver under artificially induced perfusion. CT as well as ultrasound images are similar to images in a real patient scenario. Up to our knowledge, this is the first report of a multi modality imaging experiment on real liver tissue which leads to ground truth for quantitative evaluation of registration outcomes. The images resulting from these experiments will be used for the validation of a registration approach based on a mass-spring model of the liver vasculature and vessel centreline points extracted automatically from ultrasound images. The limitation of the current setup is the reduced visibility of small vessels in the contrast-enhanced CT. Future experiments will use pulsatile contrast injection in order to avoid this effect.

\section{References}

1. Lange T, Lamecker H, Hühnerbein M, et al. Validation Metrics for Non-Rigid Registration of Medical Images containing Vessel Trees. Procs BVM. 2008; p. 8286.

2. Bao P, Warmath J, Galloway R, et al. Ultrasound-to-computer-tomography registration for image-guided laparoscopic liver surgery. Surg Endosc. 2005;19:424-429.

3. Weber S, Markert M, Lueth TC. Surface tracking of organs for registration in soft tissue surgery. In: Image Guidance and Computer Assistance for Soft-Tissue Interventions IGSTI; 2008. p. 65-74.

4. Müller SA, Maier-Hein L, Mehrabi A, et al. Creation and establishment of a respiratory liver motion simulator for liver interventions. Med Phys. 2007;34:4605-8.

5. Dagon B, Baur C, Bettschart V. Intraoperative update of liver surgery planning based on ultrasound images. In: Computer-Aided Medical Interventions: Tools and Applications. Sauramps Medical; 2007. p. 419-423. 